\title{
UK regional paediatric home parenteral nutrition (HPN) data suggest a national underestimate in service requirements
}

\author{
A. R. Barclay ${ }^{1}$, C. E. Paxton ${ }^{1}$, J. Livingstone ${ }^{2}$, D. Hoole ${ }^{3}$, F. Munro ${ }^{4}$, P. Gillett ${ }^{1}$ and D. C. Wilson ${ }^{1,5}$ \\ Departments of ${ }^{1}$ Paediatric Gastroenterology and Nutrition, ${ }^{2}$ Dietetics, ${ }^{3}$ Pharmacy and ${ }^{4}$ Surgery, Royal Hospital for Sick \\ Children, Edinburgh EH9 $1 \mathrm{LF}$, and ${ }^{5}$ Child life and Health, University of Edinburgh EH9 $1 U W$
}

Receiving HPN is the optimum standard of care for paediatric patients with intestinal failure (IF) requiring long-term parenteral nutrition (PN). Recurrent bacterial sepsis and IF-associated liver disease (IFALD) contribute to long-term morbidity and mortality in paediatric IF. Patients with irreversible IF or severe IFALD are indicated for referral to combined small-bowel-liver transplantation (CBSLT) services. Isolated liver transplantation (ILT) also has role in such patients. Nationally obtained data on paediatric IF suggest that long-term survival is above $90 \%^{(1)}$, that about fifteen paediatric patients will be commenced on HPN nationally per year ${ }^{(2)}$ and that seventeen will be indicated for referral to UK CBSLT services per year ${ }^{(3)}$.

The Royal Hospital for Sick Children Edinburgh (RHSCE) Nutrition Support Team services 1.25 million people, $2.1 \%$ of the UK population. Due to its unique service set-up (single neonatal surgical unit, single neonatal intensive care unit supplying PN beyond term, single IF nutrition support service with involvement from outset of disease), we can be confident of complete ascertainment of early-onset severe IF within the region. A retrospective survey of patients referred to the team over an 8-year period was performed (March 1997June 2005). Data obtained included demographics, diagnosis, surgery, bowel length, ileocaecal valve (ICV) removal, PN, septic episodes, central line removal, IFALD, referral for transplantation, enteral adaptation, survival, death and organ transplantation. Comparisons to national data were extrapolated using the formula $(n \times 100 / 2.1 \%)$ per 8 years.

Twenty-three patients were referred over 8 years, with all but three being PN dependent from birth. Diagnoses included short bowel syndrome (SBS; $n$ 18), neuromuscular abnormalities $(n 4)$ and congenital enterocyte disorder ( $n$ 1). A total 12696 days of PN were delivered with 314 confirmed episodes of sepsis. Central lines ( $n$ 144) were required at a mean of 88.6 patient days per line. Thirteen out of twenty three $(56 \%)$ of patients received HPN, ten $(77 \%)$ of which had SBS. Ten patients $(44 \%)$ achieved enteral adaptation at a mean age of 25.3 months. IFALD occurred in seventeen (73\%) patients, with $12(56 \%)$ being indicated for referral to CBSLT services. Ten patients were referred to CBSLT services with five patients being transplanted (three CBSLT, two ILT). Overall mortality was $44 \%$. A significant predictor for non-survival in the SBS group was residual bowel $<40 \mathrm{~cm}(28 \% v .82 \%, P=0.049)$. Extrapolated annual national rates of HPN registry and indication of referral to CBSLT services were 46.4 and 42.7 respectively in comparison with $14.6^{(2)}$ and $17.3^{(3)}$ from national surveys.

The proportion of paediatric HPN IF patients with SBS is rising, with the proportion of our patients being higher still $\left(77 \% v .40 \%{ }^{(2)}\right)$. Extreme preterm infants surviving surgical resection for necrotising enterocolitis (NEC) are at particular risk for sepsis and IFALD, impacting on needs for CBSLT and ILT. Local factors (high incidence of NEC) may in-part contribute to these figures, but they are so far in excess of nationally collected data that they strongly suggest that nationally, patients are lost due to early death, discontinuation of care or non-referral, prior to reaching tertiary paediatric gastroenterology and transplantation services. Such data may be of importance when counselling parents and for future planning of regional IF and national transplant services. Ongoing national surveillance should consider regional service design when trying to determine prevalence of such disease.

1. Goulet O, Ruemmele F, Lacaille F \& Colomb V (2004) J Pediatr Gastroenterol Nutr 38, 250-269.

2. BANS report. Artifical nutrition support in the U.K. 2000-2005. www.bapen.org.uk/pdfs/bans_reports/bans_report_05.pdf

3. Gupte G, Beath SV, Protheroe S, Murphy MS, Davies P, Sharif K, McKiernan PJ, de Villede Goyet J, Booth IW \& Kelly DA (2006) Arch Dis Child 92, $147-151$. 